\title{
Three new childhood anxiety scales have comparable reliability and validity to traditional diagnostic scales
}

Muris $P$, Merckelbach H, Ollendick T et al. Three traditional and three new childhood anxiety questionnaires: their reliability and validity in a normal adolescent sample. Behaviour Research and Therapy 2002 July; 40: 753-72.

\author{
QUESTION: Are newer childhood scales valid and reliable for assessing anxiety in \\ adolescents?
}

\section{Design}

Comparison of diagnostic scales (blinding not specified).

\section{Setting}

One secondary school in Belgium

\section{Participants}

521 adolescents; mean age 15 years (range $12-18$ years); $54 \%$ female.

\section{Description of tests}

All participants completed 6 questionnaires in their classrooms; 3 new (Multidimensional Anxiety Scale for Children (MASC); Screen for Child Anxiety Related Emotional Disorders (SCARED); Spence Children's Anxiety Scale (SCAS)), and three traditional (State-Trait Anxiety Inventory for Children (STAIC); Revised Children's Manifest Anxiety Scale (RCMAS); Fear Survey Schedule for Children - Revised (FSSC-R)). The Children's Depression Inventory (CDI) was administered first, followed by alternating new and traditional questionnaires. The order of questionnaires was counterbalanced across classrooms. Questionnaires were in Dutch, the participants' native language.

\section{Main outcome measures}

Reliability (internal consistency); correlations between new and traditional questionnaires.

\section{Main results}

All scales were reliable and internally consistent. Anxiety questionnaire scores were highly correlated. The strongest correlations were between total STAIC and RCMAS scores (correlation=0.88); SCARED and SCAS scores (correlation $=0.84$ ), and subscales measuring specific types of symptoms such as worry, social anxiety and panic. All questionnaires were correlated with the depression index (correlations between 0.5 and 0.74 ).

\section{Conclusions}

The new childhood anxiety questionnaires had comparable reliability and validity to traditional measures for a non-referred sample of adolescents.

Correlations between new and traditional anxiety questionnaires, corrected for gender and age

\begin{tabular}{lccc} 
Total score & MASC & SCARED & SCAS \\
STAIC & 0.79 & 0.87 & 0.79 \\
\hline RCMAS & 0.76 & 0.85 & 0.76 \\
\hline FSSC-R & 0.63 & 0.72 & 0.76 \\
\hline
\end{tabular}

All correlations were significant $(p<0.001)$.

\section{COMMENTARY}

Early-onset anxiety disorders can affect psychosocial development, predispose to other psychiatric disorders, continue into adulthood and negatively affect treatment of comorbid disorders. ${ }^{1}$ In externalising disorders such as attention deficit-hyperactivity disorder and oppositional defiant disorder, self-report makes little contribution to diagnosis. In contrast, studies comparing self-report with clinical diagnoses suggest that youths are aware of and accurately report the symptoms of internalising disorders. ${ }^{2}$ Self-report instruments for the assessment of child and adolescent internalising disorders are therefore extremely important for clinicians.

Following a renewed interest in juvenile anxiety disorders, several new anxiety scales have been developed. These overcome the deficits of older scales, which were mainly a downward extension of adult scales with questionable developmental relevance. ${ }^{2}$ Early-onset anxiety symptoms can be a normal part of development. ${ }^{3}$ Scales specifically designed to explore early-onset psychopathology may more precisely address developmental factors and better discriminate people with serious or transient symptoms. Muris et al compare three questionnaires developed in the last five years with three traditional questionnaires for assessing childhood anxiety. There was substantial concordance among newer and older anxiety measures. There was also co-occurrence between anxiety and depressive symptoms. This is the rule rather than the exception in children and adolescents. Discriminating between anxiety and depressive disorders is a major challenge in childhood psychopathology. Future research should explore instruments with higher divergent validity.

It is difficult to extrapolate information from scale scores to major diagnostic systems (such as ICD-10 or DSM-IV). Easy to administer, cost effective dimensional scales are commonly used to assess anxiety in children and adolescents. These provide an index of difference from the peer group (cutoff scores based on normative data). In contrast, diagnostic interviews such as Kiddie-SADS or the Diagnostic Interview for Children and Adolescents-R (DICA-R) allow categorical diagnoses according to major diagnostic systems. These are time consuming and require specific training. There are varying degrees of agreement between dimensional scales and structured diagnostic interviews. ${ }^{4}$ Muris $e t$ al found a satisfactory association among subscales measuring specific categories of anxiety symptoms. This supports the validity of more recent instruments, such as SCAS or SCARED, which tap specific DSM-IV or ICD-10 anxiety syndromes such as obsessions and compulsions, phobias and social anxiety.

These questionnaires have been found to be effective in children and adolescents referred to psychiatry outpatient clinics. ${ }^{1}$ This study suggests that the questionnaires are also reliable for diagnosing anxiety disorders in non-referred populations. Further research is needed to explore their sensitivity to change, which would allow them to be used when describing the natural history of anxiety disorders as well as treatment outcomes.

Gabriele Masi, MD IRCCS Stella Maris, Scientific Institute of Child Neurology and Psychiatry Pisa, Italy

1 Birmaher B, Brent D, Chiappetta L et al. Psychometric properties of the Screen for Child Anxiety Related Emotional Disorders (SCARED): A replication study. J Am Acad Child Adolesc Psychiatry 1999; 38:1230-6.

2 Myers K, Winters NC. Ten-year review of rating scales. II: Scales for internalizing disorders. J Am Acad Child Adolesc Psychiatry 2002; 41: 634-59.

3 Bell-Dolan DJ, Last CG, Strauss CC. Symptoms of anxiety disorders in normal children. I Am Acad Child Adolesc Psychiatry 1990, 29: 759-65.

4 Gould MS, Bird H, Jaramillo BS.Correspondence between statistically derived behavior problem syndromes and child psychiatric diagnoses in a community sample. J Abnorm Child Psychol. 1993; 21: 287-313. 